\title{
FREE SUBGROUPS OF NORMAL SUBGROUPS OF THE MULTIPLICATIVE GROUP OF SKEW FIELDS
}

\author{
A. I. LICHTMAN
}

\begin{abstract}
Let $D$ be a skew field, $D^{*}$ its multiplicative group and $H \triangleleft D^{*}$. Then $H$ contains a noncyclic free subgroup $F$, provided that there exists a nonabelian nilpotent-by-finite subgroup $G$ of $\boldsymbol{H}$.
\end{abstract}

1. Introduction. Let $D$ be a division ring, $D^{*}$ its multiplicative group. We prove the following theorem in this paper.

THEOREM 2. Let $H$ be a normal subgroup of $D^{*}$. Let us suppose that there exists a nonabelian nilpotent-by-finite subgroup $G$ of $H$. Then $H$ contains $a$ noncyclic free group $F$.

The proof of this theorem makes essential use of Tits' result that any matrix group over a field either is soluble-by-locally finite or contains a noncyclic free group (see [1]).

We remark that it is shown in [2] that Tits' theorem is not true when the field is replaced by a skew field: there exists a finitely generated group which is not soluble-by-finite and does not contain a noncyclic free subgroup, but whose group ring can be embedded in a division ring of quotients.

2. Proposition 1. Let $D$ be a division ring which is finite dimensional over its center $Z$ and $H \triangleleft D^{*}$. Let us suppose that $H$ is a soluble-by-periodic group. Then $H \subseteq Z^{*}$.

Proof. Since $(D: Z)<\infty$ the group $H$ is isomorphic to a matrix group and hence, according to Zassenhaus' theorem, there exists a maximal unique soluble normal subgroup $R$ of $H$. Since $R$ is a characteristic subgroup of $H$ we obtain $R \triangleleft D^{*}$. Scott proved (see [3], [4]) that any soluble normal subgroup of $D^{*}$ is contained in the center. The assertion follows now from the fact that a periodic subgroup of the group $D^{*} / Z^{*}$ must be trivial (see [5]).

Lemma 1. Let $D$ be a division ring, $x, y$ two elements of $D^{*}$, such that $1 \neq z=y^{-1} x^{-1} y x, z x=x z$ and $z y=y z$. Let $Q$ be the subring of $D$ which is generated by the elements $1, z, x, y$ and $L$ be the subring of $D$ which is generated by the elements $1, z$. Then

Received by the editors January 25, 1977.

AMS (MOS) subject classifications (1970). Primary 16A40.

Key words and phrases. Multiplicative group of division ring, free group. 
(a) For any $q \in Q$ there exists a representation

$$
q=\sum \lambda_{k l} x^{k} y^{l}
$$

where $\lambda_{k l} \in L ; k=0,1, \ldots, l=0,1, \ldots$

(b) The division subring $D_{1}$ generated by the elements $x, y$ is finite dimensional over its center $T$, provided that either

$$
\sum \alpha_{i j} x^{i} y^{j}=0
$$

for some nonzero $\alpha_{i j} \in L$; or

$$
z^{n}=1
$$

for some natural $n$.

Proof. (a) Follows from the relation $y x=z x y$.

(b) Let us suppose that (2) holds. We can write it in the form

$$
\sum_{j} \beta_{j} y^{j}=0
$$

where

$$
\beta_{j}=\sum_{i} \alpha_{i j} x^{i}
$$

Let $P$ be the prime subfield of $D$ and consider the subfield $P(z, x)$ of $D_{1}$. If in (4) some $\beta_{j} \neq 0$, we obtain from (1) that $\operatorname{dim}_{l}\left(D_{1}: P(z, x)\right)$ is finite. By [6, Chapter VII, §9, Theorem 1] $D_{1}$ is finite dimensional over its center.

Similarly, if in (5) all the $\beta_{j}=0$, we obtain from this that $D_{1}$ is finite dimensional over $P(z, y)$ and hence over $T$.

Suppose now that $z^{n}=1$ for some natural $n$. We obtain $x^{n} y=y x^{n}$; as above $D_{1}$ is finite dimensional over the subfield $P\left(z, x^{n}, y\right)$ and the assertion follows.

THEOREM 1. Let $H$ be a normal subgroup of $D^{*}$ such that $H$ contains $a$ nonabelian nilpotent subgroup $G$. Then $H$ contains a noncyclic free group $F$.

Proof. Since $G$ is nilpotent, we can find two elements $x, y$ such that $1 \neq z=y^{-1} x^{-1} y x$ and $z x=x z, z y=y z$. Let $D_{1}$ and $Q$ be as in Lemma $1, P$ be the prime subfield of $D$ and $C$ the ring of integers.

Suppose first that one of the conditions (2) or (3) of Lemma 1 holds. We consider the nonabelian subgroup $H_{1}=H \cap D_{1}$, which is a normal subgroup of $D_{1}^{*}$. Proposition 1 implies that $H_{1}$ cannot be soluble-by-periodic. Hence, via Tits' theorem, $H_{1}$ contains a noncyclic free group.

We can suppose therefore that neither of the conditions (2) and (3) holds, which means, in particular, the uniqueness of the representation (1).

We suppose therefore in the sequel that the elements $x^{k} y^{l}, k=0,1, \ldots$, $l=0,1, \ldots$, are linearly independent over $L$. It can also be assumed that the subring $L$ is an infinite noetherian domain. We therefore have the following three possibilities.

I. $P$ is the field of rationals, $z$ is algebraic over $P$ and hence $L$ is isomorphic 
to some subring $C(\theta)$ of an algebraic number field.

II. $P$ is the field of rationals, $z$ is transcendental over $P$ and $L$ is the polynomial ring $C[z]$.

III. $P$ is a finite field, $z$ is transcendental over $P$ and $L$ is the polynomial ring $P[z]$.

We show that there exists some $\mathfrak{B}$-adic valuation $\rho$ of the quotient field $P(z)$ of $L$ such that the following conditions hold:

(i) The valuation ring $\tilde{L}$ of $\rho$ contains $L$.

(ii) $\rho(z)=0$ and $\rho(1-z)=0$.

(iii) If $\tilde{A}$ is the maximal ideal of $\tilde{L}$ then the quotient $\operatorname{ring} \tilde{L} / \tilde{A}$ is a finite field.

In fact, in I and III we take any $\mathfrak{B}$-adic valuation $\rho$ of $P(z)$ which satisfies (ii); it follows immediately that $\rho$ satisfies also (i) and (iii). In II we choose any maximal ideal $A$ of $L$ such that $z(1-z) \notin A$. The quotient $\operatorname{ring} L / A$ is a field and the ideal $A$ is generated by some prime $p \in C$ and some monic polynomial $f[x] \in C[x]$, which is irreducible modulo $(P)$. One can check that the powers of $A$ satisfy the conditions of [8, Chapter $18, \S 141]$ and therefore $A$ defines $\mathfrak{B}$-adic valuations of $L$ and of $P(z)$. Once more, we see that (i)-(iii) hold.

Let $\tilde{Q}$ be the subring of $D_{1}$ generated by $Q$ and $\tilde{L}$. The elements $x^{k} y^{l}$ remain linearly independent over the ring $\tilde{L}$, i.e. $\tilde{Q}=\tilde{L} \otimes_{\tilde{L}} Q$. The subring $\tilde{L}[x]$ of $\tilde{Q}$ is a polynomial ring over the noetherian domain $\tilde{L}$ and $\tilde{Q}$ is a skew polynomial ring over $\tilde{L}[x]$. Hence, $\tilde{Q}$ is a noetherian ring.

Now let $(A)=\left\{\tilde{q} \in \tilde{Q} \mid \tilde{q}=\sum a_{k l} x^{k} y^{l}, a_{k l} \in \tilde{A}\right\}$. We see that $(A)$ is an ideal in $\tilde{Q}$ and we will prove that $(A)$ is prime.

Indeed, let us assume that there exist elements $\tilde{q}_{1}$ and $\tilde{q}_{2}$ of $\tilde{Q}$ such that $\tilde{q}_{1} \tilde{q}_{2} \in(A)$ and $\tilde{q}_{i} \notin(A), i=1,2$. We order lexicographically the products

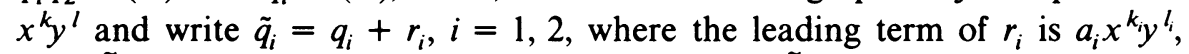
$a_{i} \notin \tilde{A}$ and all terms in $q_{i}$ have coefficients in $\tilde{A}$. Thus, $\tilde{q}_{1} \tilde{q}_{2}=q^{\prime}+r_{1} r_{2}$, where $q^{\prime} \in(A)$ and the leading term of $r_{1} r_{2}$ is $a_{1} a_{2} z^{k_{2} l_{1}} x^{k_{1}+k_{2}} y^{l_{1}+l_{2}}$. But $\tilde{q}_{1} \tilde{q}_{2} \in(A)$ implies $a_{1} a_{2} z^{k_{2} l_{1}} \in \tilde{A}$. Since $\tilde{A}$ is maximal and $z \notin \tilde{A}$, we obtain $a_{1} \in \tilde{A}$ or $a_{2} \in \tilde{A}$ which is a contradiction.

Let $M$ be the complement of $(A)$ in $\tilde{Q}$. We shall prove that the set $M$ is a right denominator set in $\tilde{Q}: M$ is multiplicatively closed (because $(A)$ is prime); for any two elements $m \in M, r \in \tilde{Q}$, there exist $m_{1} \in M, r_{1} \in \tilde{Q}$ such that

$$
r m_{1}=m r_{1} \text {, }
$$

and $r m=0$ or $m r=0$ implies $r=0$ (see [7, §0.5]).

Indeed, $\tilde{Q}$ is a noetherian ring without zero divisors and therefore has a right ring of quotients (see, for instance, [7].) Thus, two elements $m_{1}, r \in \tilde{Q}$ can be found such that (6) holds; we only need to prove that $m_{1}$ can be chosen in $M$.

Let us suppose that in (6) $m_{1} \in(A)$, i.e. $m_{1}=\sum \mu_{k l} x^{k} y^{l}, \mu_{k l} \in \tilde{A}$. Since $(A)$ is prime and $m \notin(A)$, we obtain $r_{1} \in(A)$, i.e. $r_{1}=\sum \gamma_{r s} x^{r} y^{s}, \gamma_{r s} \in \tilde{A}$. Moreover, let $\pi$ be any element of the ideal $\tilde{A}$ such that $\rho(\pi)=1$, and $\pi^{n}(n \geqslant 1)$ is 
the greatest common divisor of the elements $\mu_{k l}$, i.e. $m_{1}=\pi^{n} m^{\prime}$, where $m^{\prime} \in M$. From (6) we now obtain that $\pi^{n}$ is a common divisor of the elements $\gamma_{r s}$. Hence, cancelling $\pi^{n}$ from both sides of (6), we obtain

$$
r m^{\prime}=m r^{\prime}, \quad m^{\prime} \in M, r^{\prime} \in \tilde{Q},
$$

which means that $M$ is a right denominator set in $\tilde{Q}$.

Consider now the right quotient ring $\tilde{Q}_{M}$ of $\tilde{Q}$ with respect to $M$. Any element $h \in \tilde{Q}_{M}$ has the form $h=(\tilde{q}, m)$ where $\tilde{q} \in \tilde{Q}, m \in M$, and we see that $h$ is invertible in $\tilde{Q}_{M}$ iff $\tilde{q} \in M$. This implies that the set $B$ of noninvertible elements of $\tilde{Q}_{M}$ is an ideal, and $B \cap \tilde{Q}=(A)$; moreover, the quotient ring $\bar{Q}=\tilde{Q}_{M} / B$ is a division ring.

Now let $\tilde{H}=H \cap \tilde{Q}_{M}$. We see that $\tilde{H}$ is a normal subgroup of the group of units of $\tilde{Q}_{M}$. If $\bar{H}$ means homomorphic image of $\tilde{H}$ under the natural homomorphism $\varphi$ of $\tilde{Q}_{M}$ onto $\bar{Q}$, we obtain from the fact that $\tilde{Q}_{M}$ is a local ring, that $\bar{H}$ is a normal subgroup of the group $\bar{D}^{*}$.

We prove now that $\bar{H}$ contains a noncyclic free $\operatorname{subgroup} N$; this fact will imply that $\tilde{H}$ contains a noncyclic free subgroup $F$.

Indeed, let $\bar{x}=\varphi(x), \bar{y}=\varphi(y)$ and let $K$ be the division subring of $\bar{Q}$ generated by the elements $\bar{x}, \bar{y}$. We have

$$
\bar{y} \bar{x}=\bar{z} \bar{x} \bar{y} ; \quad \bar{z}=\varphi(z) .
$$

On the other hand, the linear independence of the elements $x^{k} y^{l}$ over $L$ implies that $(A) \cap \tilde{L}=\tilde{A}$; hence $B \cap \tilde{L}=(B \cap \tilde{Q}) \cap \tilde{L}=(A) \cap \tilde{L}=\tilde{A}$. The choice of the valuation $\rho$ now implies that $\bar{z}$ is a nonidentity element of finite order. By Lemma $1 K$ is finite dimensional over its center.

Finally, let $\bar{H}_{1}=\bar{H} \cap K^{*}$. Then $\bar{H}_{1} \triangleleft K^{*}$ and $\bar{x}, \bar{y} \in \bar{H}_{1}$. Since $\bar{z} \neq 1, \bar{H}_{1}$ is a nonabelian group. Proposition 1 now implies that $\bar{H}_{1}$ cannot be soluble-by-periodic and, according to Tits' theorem, $\bar{H}_{1}$ contains a noncyclic free group $N$. As was mentioned above, this implies that there exists a noncyclic free group $F \subseteq \tilde{H}$, which completes the proof.

3. Proof of Theorem 2. Let $G$ be a nonabelian subgroup of $H$ for which there exists a nilpotent normal subgroup $G_{1}$ of $G$ such that $G / G_{1}$ is finite. By Theorem 1, it may be supposed that $G_{1}$ is abelian.

We consider now the division subring $D_{1}$, which is generated by $Z$ and $G$. If now $Z\left(G_{1}\right)$ means the subfield generated by $G_{1}$ and $Z$, we obtain, as above, that $\operatorname{dim}_{l}\left(D_{1}: Z\left(G_{1}\right)\right)$ is finite and $D_{1}$ is finite dimensional over its center.

Let $H_{1}=H \cap D_{1}$. Then $H_{1} \triangleleft D_{1}^{*}$ and $G \subseteq H_{1}$. Hence $H_{1}$ is nonabelian and therefore cannot be soluble-by-finite; as above, we conclude that there exists a noncyclic free group $F \subseteq H_{1}$.

ACKNOWLEDGEMENT. I am grateful to the referee and to the editor for their useful remarks.

\section{REFERENCES}

1. J. Tits, Free subgroups in linear groups, J. Algebra 20 (1972), 250-270.

2. A. I. Lichtman, On subgroups of the multiplicative group of skew fields, Proc. Amer. Math. Soc. 63 (1977), 15-16. 
3. W. R. Scott, On the multiplicative group of a division ring, Proc. Amer. Math. Soc. 8 (1957), 303-305.

4. Group theory, Van Nostrand, Princeton, N. J., 1965.

5. A. I. Lichtman, On the normal subgroups of the multiplicative group of a skew field, Dokl. Akad. Nauk SSSR 152 (1963), 812-815 = Soviet Math. Dokl. 153 (1963), 1424-1429.

6. N. Jacobson, Structure of rings, Amer. Math. Soc. Colloq. Publ., vol. 37, Amer. Math. Soc., Providence, R. I., 1956.

7. P. M. Cohn, Free rings and their relations, Academic Press, New York, 1971.

8. B. L. Van der Waerden, Algebra. I, Springer-Verlag, Berlin and New York, 1971.

Department of Mathematics, Ben Gurion University of THE Negev, BeER-SheVA, ISRael 